\title{
Epistemische Dreiecksbeziehungen
}

\author{
Überlegungen zur Ko-Konstruktion von Krankheit,
} Individuum und Gesellschaft

\section{STEFAN BECK}

Vor einigen Jahren veröffentlichte eine der international führenden Medizinanthropologinnen, die in Montreal lehrende Margaret Lock, einen in der Anthropologie viel beachteten Artikel unter dem Titel »Das Verschwinden des Gens und die Rückkehr der Wahrsagerei«. Ihr Kernargument: Der seit Beginn des 20. Jahrhunderts in der Biologie und Medizin dominierende genetische Determinismus sei tot; neue Erklärungsmodelle, vor allem die Entdeckung epigenetischer Mechanismen und epigenetischer Plastizität in Reaktion auf chemische Umwelteinflüsse und - noch wichtiger - auf soziale Interaktionsprozesse, seien dabei, einfache mechanistische Erklärungsmodelle zu verdrängen, die in der zweiten Hälfte des 20. Jahrhunderts die Biologie dominiert hätten:

»Die Entdeckung, dass individuelle Entwicklung, Altern und die Umwelt die Aktivität [der Zelle] auf molekularem Niveau mit prägen, hat das Konzept eines genetisch vorherbestimmten Körpers beseitigt und an seine Stelle eine wesentlich fluideres, schwer fassbares Objekt gesetzt. Organismen sind offensichtlich mehr als die Summe ihrer [molekularen] Teile und es ist nun nicht mehr zu bestreiten, dass Gene sehr wenig, wenn überhaupt, determinieren und lediglich als Akteure [unter anderen] in einem außergewöhnlich komplexen Geschehen anzusehen sind. ${ }^{1}$

1 Margaret Lock: »Eclipse of the Gene and the Return of Divination«, in: Current Anthropology 46 Supplement (2005), S. S47-S70, hier S. S50; Übers. SB. 
Gemäß diesen neueren Modellen folge der Körper keinem starren genetischen Programm mehr, sondern reagiere dynamisch auf Außeneinflüsse. Hierdurch sei die Hoffnung, dass immer mehr genetische Tests eine immer größere Vorhersagekraft für die individuelle Krankheitsentwicklung und Gesundheitserwartungen ermöglichten, empfindlich eingeschränkt. Genetische Diagnostik grenze in vielen Fällen daher eher an Wahrsagerei als an wissenschaftlich exakte Prognose. Konkret arbeitet Lock dies an den genetischen Tests heraus, die momentan zur Prädiktion von Alzheimer-Demenz angewandt werden und die nur eine geringe Vorhersagekraft, und dies auch nur in sehr spezifischen Populationen, besitzen.

Wichtiger ist für Lock jedoch, dass die Ergebnisse epigenetischer Forschungen nicht nur die in den Sozialwissenschaften spätestens seit dem Streit um soziobiologische Erklärungsansätze in den 1960er Jahren fest etablierte Trennung von Natur I Kultur infrage stellen - hierauf komme ich gleich noch ausführlicher zurück -, sondern sie seien auch als fundamentale Herausforderung an die Sozialwissenschaften zu verstehen: Wenn die Befunde der Epigenetik zuträfen, dass Verhaltensweisen und soziale Interaktionsmuster einen Einfluss auf die Regulation und Funktion des Genoms besäßen, dann müssten Anthropologen beginnen, ihre Methoden, ihre kulturvergleichende Perspektive und ihre theoretischen Ansätze auch dazu zu nutzen, Einsichten in jene üblicherweise als sozial und kulturell verstandenen Faktoren zu gewinnen, die krankheitsvermeidende oder krankheitsgenerierende Effekte haben könnten. Und Anthropologen sollten diese Befunde auch offensiv in den durch naturwissenschaftliche Perspektiven dominierten medizinischen Diskurs einbringen. Was Lock hiermit fordert, ist nichts weniger als eine gänzlich neue Rolle für die Medizinanthropologie oder die Medizinsoziologie im Verhältnis zur Biomedizin, die auch die Entwicklung neuer Formen der Kooperation zwischen Sozial- und Naturwissenschaften erfordert. Um es ein wenig zuzuspitzen: Statt wie bisher vor allem die Medizin als moderne Institution und ihre Machteffekte zu untersuchen oder deren Wirkungen im Alltag, den Umgang von Patienten mit neuen Krankheitskonzepten und medizinischen Interventionen zu analysieren, sei nun von den Sozialwissenschaften gefordert, substanziell zu den Erkenntnisprozessen der Medizin und Biologie selbst beizutragen. Damit ist nichts weniger als die für anthropologische Konzeptionen durchaus weitreichende Frage aufgeworfen, ob Belege dafür gefunden werden können, dass nicht nur die menschliche Natur Kultur prägt, sondern auch umgekehrt menschliche Kultur, soziale Interaktionsmuster und Lebensweisen die individuelle wie kollektive menschliche Natur beeinflussen - und wenn dies belegbar wäre, welche Mechanismen hierfür verantwortlich gemacht werden können. 


\section{FragePRogramm UND EINIGE NOTWENDIGE REVISIONEN}

Im Folgenden soll gefragt werden, welche Vorstellungen des Sozialen und welche Konzepte des Individuums in verschiedenen Krankheitskonzepten impliziert sind. Dazu werden einige Überlegungen dazu vorgestellt, wie die Medizin den Zusammenhang zwischen Umwelt und Körper, zwischen Pathologischem und Sozialem in ihrer Fachgeschichte konstruiert hat. An drei Beispielbereichen psychosomatischen Erkrankungen, stressbedingten pathologischen Veränderungen und schließlich epigenetischen Theorien der Krankheitsentstehung - wird verdeutlicht, wie medizinische Praktiken zu einer Ko-Konstruktion des Pathologischen und des Sozialen beitragen. ${ }^{2}$ Im Kern geht es bei vielen Krankheitskonzepten darum - so die hier vertretene These -, Mechanismen zu identifizieren, durch die das Soziale und das Kulturelle >unter die Haut gehen< und dort pathologische Prozesse auslösen. Mit diesen drei Grabungsschnitten durch die Sedimente der medizinischen Fachgeschichte soll dabei angedeutet werden, dass Krankheitskonzepte aus zwei Gründen stets durch eine zeit- und kulturspezifische Sicht auf Gesellschaft und Individuen geprägt sind: erstens, weil in den Ätiologien der Zusammenhang von Lebensumständen und Krankheitsentstehung thematisiert wird; und zweitens, weil Heilungs- und Präventionsoptionen stets Interventionen in soziale Lebensumstände ebenso implizieren wie normative Vorstellungen darüber, wie ein Individuum oder ein Kollektiv präventiv handeln sollte. Aus einer ethnologischen Perspektive ist diese historisch sehr unterschiedlich gehandhabte Dreiecksbeziehung zwischen Krankheit - Gesellschaft - Individuum vor allem deshalb interessant, weil medizinische und biowissenschaftliche Modelle das Wissen, das Menschen in modernen Gesellschaften über sich und die Gesellschaften haben, in denen sie leben, in entscheidender Weise mitprägen.

Ein solches Frageprogramm erfordert die Revision üblicher theoretischer Reflexe, die in den Sozialwissenschaften seit geraumer Zeit gegenüber den Natur-

2 Im Folgenden können diese Zusammenhänge nur angedeutet werden; sie bedürfen einer wesentlich intensiveren Auseinandersetzung und der sorgfältigen Kontextierung in die jeweiligen - nicht nur wissenschaftlichen - historischen Debatten und den je herrschenden Stand wissenschaftlicher Erkenntnisproduktion: vgl. hierzu Lorraine Dastons systematische Überlegungen zur Rolle der Wissenschaftsgeschichte bei der Befremdung der Standard-Narrative der Wissenschaftsentwicklung durch die ReKontextierung wissenschaftlicher Praktiken und Denkstile in die relevanten raumzeitlichen Koordinaten: »Science Studies and the History of Science«, in: Critical Inquiry 35 (2009), S. 798-813. 
wissenschaften vorherrschen. Der eingangs zitierte Artikel von Margaret Lock ist als ein wichtiger wissenschaftspolitischer Aufruf an die Anthropologie zu lesen, eine solche Revision einzuleiten. Denn Lock fordert nichts weniger, als die seit den 1960er Jahren in den Sozialwissenschaften generell und in der Anthropologie speziell aufkommende »Bio-Phobie« zu überwinden. ${ }^{3}$ Hintergrund ist, dass die Anthropologie sich in ihrer langen, weit in das 19. Jahrhundert zurückreichenden Fachgeschichte immer auch mit der biologischen Materialität des Körpers befasst hatte, diese Kompetenz jedoch im 20. Jahrhundert zunehmend aufgab. In Deutschland geriet die physische Anthropologie schon in den 1920er Jahren zunehmend unter den Einfluss rassistischen Denkens und wurde nach 1945 mit guten Gründen abgewickelt; als »medizinische Anthropologie« wurde sie in die Peripherien oder Kellergeschosse der Universitätskliniken verbannt. Dagegen konnte sie sich vor allem in Nordamerika als nicht-essenzialistisch argumentierende Disziplin erhalten. So bestehen bis heute an einigen Universitäten integrativ angelegte Institute, an denen Anthropologen mit kultureller, linguistischer, archäologischer und eben auch biologischer Spezialisierung Seite an Seite arbeiten. Dieser four field approach genannte Ansatz verfolgt den alten anthropologischen Anspruch, dem »ganzen Menschen « in seiner historischen Entwicklung und kulturell diversen Gegenwart gerecht zu werden. ${ }^{4}$

Doch das Teilgebiet der physical anthropology wurde seit den 1960er Jahren auch an nordamerikanischen Universitäten immer stärker der Kritik kulturtheoretisch ausgerichteter Anthropologen unterzogen, ein Konflikt, der schließlich zur Spaltung zahlreicher Institute führte. Beschleunigt wurde dieser Ausdifferenzierungsprozess nochmals in den letzten 20 Jahren, als die physical anthropology zunehmend begann, auch auf molekulargenetische Methoden zurückzugreifen und sich an neo-evolutionistischen Theorien $\mathrm{zu}$ orientieren. Viele sich eher >links< verortende, vor allem aber feministisch orientierte AnthropologInnen kritisierten, dass durch die Übernahme biologischer Befunde genetischdeterministische Denkstile und damit ein gefährlicher naturalistischer Fatalismus im Blick auf den Menschen und seine Entwicklungspotenziale in der Anthropologie Einzug halten. Eine solche Perspektive sei nur mit konservativen politischen Ideologien, nicht jedoch mit dem Emanzipationsanspruch der Sozialwissenschaften kompatibel. Margaret Locks Aufruf steht noch ganz in der Tradition dieser Kritiklinie - sie weist jedoch insofern darüber hinaus, als sie argumentiert,

3 Vgl. hierzu Ted Benton: »Biology and Social Science: Why the return of the repressed should be given a (cautious) welcome«, in: Sociology 25 (1991), S. 1-29.

4 Vgl. hierzu ausführlich Stefan Beck: »Natur | Kultur. Überlegungen zu einer relationalen Anthropologie«, in: Zeitschrift für Volkskunde 104 (2008), S. 161-199. 
dass die naturwissenschaftliche Diskussion nun selbst die Unhaltbarkeit des von Anthropologen so lange kritisierten genetischen Determinismus anerkenne. Hierzu verweist sie zutreffend auf die Befunde der Epigenetik oder der Systembiologie.

Problematisch an der in der internationalen Anthropologie dominierenden Sicht der Dinge ist dabei vor allem, dass damit eine in den Sozialwissenschaften verbreitete, verkürzte Sicht auf den biologischen Forschungsstand fortgeschrieben wird, eine Sicht, die sich eher aus der Rezeption populärwissenschaftlicher Programmatiken denn aus der intensiven Auseinandersetzung mit Forschungspraxen und theoretischen Überlegungen der Biologie speist. Denn abgesehen von einigen wenigen Exponenten genetisch-deterministischen Denkens - wie etwa Edward O. Wilson, der in den 1970er Jahren das Konzept der »Soziobiologie « propagierte, ${ }^{5}$ Richard Dawkins, der mit seinem Buch »Das egoistische Gen« eine neo-darwinistische Theorie der Kultur vorlegte, ${ }^{6}$ und einer Reihe von »evolutionären Psychologen«, die auch die kulturelle Entwicklung des Menschen vollständig mit theoretischen Ansätzen der Evolutionstheorie erklärt sehen wol$\operatorname{len}^{7}$ - setzte sich ein solcher starker Reduktionismus oder ein molekularer Determinismus, der menschliches Verhalten und Kultur allein auf die Wirkungsweise von Genen zurückführen wollte, nie vollständig in der Biologie durch. Verkürzt gesagt kann die Geschichte der Biologie und Medizin nur unvollständig als eine der zunehmenden Ausdehnung der Molekularisierung geschrieben werden, wenn darunter verstanden wird, dass Methoden und Theorien der Chemie und Physik in immer größerem Umfang für die Erklärung physiologischer Prozesse herangezogen wurden. Die seit Mitte des 20. Jahrhunderts immer bedeutendere Rolle der Genetik scheint reduktionistische medizinische Modelle der Krankheitsentstehung zu befördern. Allerdings muss gleichzeitig berücksichtigt werden, dass das Rätsel des dynamischen Zusammenhanges von Organismen und ihren Umwelten stets eines der zentralen, nicht einfach biochemisch zu reduzierenden Probleme der Biologie und Medizin darstellte, eine Problematik, auf die gerade die Genetik keine ausreichende Antwort bereitstellt. Und ebenso erweist sich gegen den Verdacht, dass zunehmend eine Ausdehnung der naturwissenschaftlich informierten Biomacht zu beobachten sei, der Hinweis als an-

5 Edward O. Wilson: Sociobiology. The New Synthesis, Cambridge, MA, London 1975.

6 Richard Dawkins: Das egoistische Gen, Reinbek bei Hamburg 1995.

7 Vgl. etwa Alex Mesoudi/Andrew Whiten/Kevin N. Laland: »Towards a unified science of cultural evolution«, in: Behavioral and Brain Sciences 29 (2006), S. 329347. 
gebracht, dass die biologische Existenz des Menschen durch eine Vielzahl sehr unterschiedlicher - und nicht nur naturwissenschaftlich fundierter - Machttechnologien geprägt ist. ${ }^{8}$

Folgt man der Argumentation des französischen Wissenschaftshistorikers Georges Canguilhem, dann wurde die zentrale Frage der Biowissenschaften nach dem dynamischen Zusammenhang von Umwelt und Organismus seit dem ausgehenden 18. Jahrhundert zunehmend als Problem der biologischen Regulation gefasst. Für Canguilhem ist die Geschichte der Wissenschaften vom Leben dabei durch eine zunehmend systemischere Sicht auf den Körper charakterisiert, die paradoxerweise - gerade durch die immer detaillierteren, mit reduktionistischen Methoden produzierten Einsichten über die physikalischen und chemischen Grundlagen von Lebensprozessen erforderlich sei. Hier kann nicht näher auf seine Rekonstruktion des Wandels der Denkstile in den Lebenswissenschaften eingegangen werden, entscheidend ist aber seine Beobachtung, dass die Lösungsvorschläge, die Mediziner und Biologen für das Problem der Regulation entwickelten, auf Konzept-Importe angewiesen waren: Im 18. und frühen 19. Jahrhundert auf mechanistische Modelle, bei denen der Körper als Maschine verstanden wurde; ab Mitte des 19. Jahrhunderts auf ökonomische und politische Modelle des Sozialen, um Prozesse der Selbstorganisation (zum Beispiel in der Embryonalentwicklung) und bei der Integration zellulärer und organischer Funktionen zu verstehen. Mit dem späten 19. Jahrhundert sieht Canguilhem dann eine Entwicklung einsetzen, bei der die Biologie das Stadium analogen Theoretisierens aufgebe und sie eigenständige Modelle entwickle: Der Organismus werde »zu seinem eigenen Modell«, es entstehe ein genuin biologisches Modell der Organisation heterogener Teile. ${ }^{9}$ Entscheidend ist, dass ab diesem Zeitpunkt die Biologie nicht mehr auf einen Konzept-Import angewiesen zu sein scheint, sondern ihre Konzepte auch erfolgreich exportieren kann: Nicht mehr die Maschine oder die Gesellschaft diene als Modell für den Organismus, sondern Maschinen und Gesellschaften könnten von Sozialwissenschaftlern nun wie Organismen gedacht werden. Die bereits erwähnten Ansätze der Soziobiologie oder der Evolutionären Psychologie sind hier gleichsam nur die Endmuräne einer Entwicklung, die aus Sicht der Sozialwissenschaften nichts anderes darzustellen scheint als einen epistemischen Imperialismus der Naturwissenschaften, der entschieden abzuwehren ist.

8 Vgl. Sujatha Raman/Richard Tutton: »Life, Science, and Biopower«, in: Science, Technology \& Human Values (2009), S. 1-24.

9 Georges Canguilhem: A vital rationalist. Selected writings from Georges Canguilhem, New York 2000, S. 302; Übers. SB. 


\section{EpISTEMische DreiecksbezieHUNGeN}

Im weiteren Verlauf des Artikels sollen diese Anregungen Canguilhems mit einer veränderten - und wesentlich bescheideneren - Frage aufgenommen werden. Gefragt werden soll nicht nach den wechselnden Leitdiskursen, mit denen in der Medizin die Frage nach der Regulation des Zusammenhanges von Organismen und ihren Umwelten bzw. die Frage von Krankheitsentstehung je verschieden beantwortet wurde. Stattdessen wird gefragt, welche Vorstellungen des Sozialen und welche Konzepte des Individuums in unterschiedlichen Krankheitskonzepten impliziert sind. Dabei sind zwei Argumentationsmuster voneinander zu unterscheiden: einerseits die in den Sozialwissenschaften weitgehend unkontroverse und durch die Medizin nicht bestrittene Annahme eines konstruktivistischen Zusammenhanges zwischen Krankheitsklassifikation und sozialsomatischen Verhaltensänderungen bei den so Klassifizierten. Davon zu unterscheiden ist andererseits die weiter gehende Annahme, dass eine kausale Wirkung von sozialen auf physische Phänomene ausgehen könnte; hier geht das Soziale direkt unter die Haut.

Die konstruktivistische These, bei der die Sozialwissenschaften gleichsam auf der >sicheren Seite< stehen, wird etwa dann relevant, wenn jemand mit der konkreten Diagnose konfrontiert wird, er leide an einem stressbedingten metabolischen Syndrom, das für einen hohen Blutdruck und einen a-normalen Fettstoffwechsel verantwortlich sei und das Risiko steigere, frühzeitig an Diabetes zu erkranken. ${ }^{10}$ Für die Medizinsoziologin Gayle Sulik wird spätestens in dem Augenblick, in dem eine so Diagnostizierte anfängt, auf diese Diagnose positiv durch Umstellung ihres Lebensstiles zu reagieren, eine »technoscientific illness identity « geschaffen $^{11}$ - das heißt, die Patientin macht sich die aktuell geltenden medizinischen Annahmen über die Ätiologie des hohen Blutdrucks zu eigen und versucht, die empfohlenen Konsequenzen zu realisieren: ihren Lebensstil zu ändern. Aber auch für Menschen, die nicht selbst von einer konkreten Diagnose betroffen sind, ist es mehr als wahrscheinlich, dass sie durch Präventionskampagnen, popularisierte medizinische Erkenntnisse oder schlicht Alltagskommunikation mit Theorien darüber konfrontiert werden, welche ihrer Lebensstil-

10 Vgl. Jörg Niewöhner: »Die zeitlichen Dimensionen von Fett - Körperkonzepte zwischen Prägung und Lebensstil«, in: Jörg Niewöhner/Christoph Kehl/Stefan Beck (Hg.), Wie geht Kultur unter die Haut? Emergente Praxen an der Schnittstelle von Medizin, Lebens- und Sozialwissenschaft, Bielefeld 2008, S. 113-142.

11 Gayle A. Sulik: »Managing biomedical uncertainty: the technoscientific illness identity«, in: Sociology of Health \& Illness 30 (2009), S. 1-18. 
Entscheidungen gesundheitlich »riskant « sind. Für den britischen Sozialwissenschaftler Nikolas Rose entsteht durch dieses verallgemeinerte »risk thinking « eine neue Form der Subjektivität in der Moderne. Dieses »Risikodenken« verdränge ältere Konzepte gesundheitlicher Veranlagung, aktualisiere potenzielle Zukünfte in der Gegenwart und mache sie zum Objekt rationaler Kalkulation: »Hence new forms of life are taking shape [...], along with new individual and collective subjectifications of those $>$ at risk $<$, and, of course, new extensions of the power of expertise to all who are now understood as >pre-patients $<.{ }^{12}$ Diese moderne Figur des Prä-Patienten ist für Rose Objekt ebenso wie Subjekt einer neuartigen »Politik des Lebens«, die vor allem durch Praktiken der »Gouvernementalität « charakterisiert sei: einem Modus der Intervention und Regulation in der späten Moderne, der nicht mehr durch Repression, sondern vor allem durch neue Subjektivierungsformen operiere, nämlich durch den normalisierten Anspruch an das moderne Subjekt, nach wissenschaftlichen Standards zur rationalen Sorge um sich beizutragen. ${ }^{13}$

Was Rose hier in einer an Michel Foucault orientierten Theorietradition analysiert, weist Parallelen auf zu der These des kanadischen Wissenschaftsphilosophen Ian Hacking, dass Klassifikationspraktiken - etwa wenn durch die Medizin körperliche Symptome zum Zeichen eines Syndroms werden und deren Träger entsprechend als »erkrankt an X« klassifiziert werden - auf Selbstverständnisse und Handlungsmuster der Klassifizierten zurückwirken. Hacking nennt dies »looping effect«, seine Perspektive auf solche realen Effekte durch Klassifikationspraktiken »dynamischen Nominalismus «: Hacking fragt, wie in spezifischen historischen Situationen »Wissensobjekte« konstituiert werden und »wie diese verschiedenen Konzepte, Praktiken und korrespondierenden Institutionen, die wir als Wissensobjekte behandeln können, neue Wahl- und Handlungsoptionen für Menschen eröffnen ${ }^{14}{ }^{14}$

Was bei einer Grippe noch vernachlässigbar erscheint, ist bei komplexeren psychologischen Syndromen, wie sie von Hacking thematisiert werden - etwa der multiplen Persönlichkeitsstörung oder der Posttraumatischen Belastungsstörung - alles andere als trivial: Hier ist damit zu rechnen, dass eine wissenschaftliche Klassifikation die Selbstwahrnehmung der Patienten entscheidend modifiziert. In dem im Folgenden diskutierten Zusammenhang geht es um etwas, was

12 Nikolas S. Rose: Politics of life itself: biomedicine, power, and subjectivity in the twenty-first century, Princeton 2007, S. $19 f$.

13 Vgl. Ulrich Bröckling/Susanne Krasmann/Thomas Lemke: Gouvernementalität der Gegenwart. Studien zur Ökonomisierung des Sozialen, Frankfurt/.M. 2001.

14 Ian Hacking: Historical Ontology, Cambridge 2002, S. 2; Übers. SB. 
ich einen erweiterten Looping-Effekt nennen möchte. Während Hacking fragt, wie Krankheitsklassifikationen die individuelle Wahrnehmung des Körpers transformieren, steht im Folgenden im Zentrum, wie die in Krankheitsdefinitionen implizierte Ko-Konstruktion von Krankheit - Gesellschaft - Individuum zusätzlich die Selbstbeobachtung von Gesellschaft beeinflusst und welche Rekonfigurationen etwa der moralischen Ansprüche an das Individuum damit verbunden sind.

Um diesen Zusammenhang nochmals klarer zu fassen, ist ein kurzer Exkurs in die medizinanthropologische Debatte nützlich. Für die medical anthropology etablierte der in Harvard lehrende Arthur Kleinman Mitte der 1970er Jahre ${ }^{15}$ die Unterscheidung zwischen drei Begriffen: Disease, also Krankheit, bezeichnet bei Kleinman erstens die vor allem von der Medizin diagnostizierten »Abnormalitäten « in der Struktur oder der Funktion von Organen und Organsystemen. Dabei kann es sich durchaus um »pathologische Zustände« handeln, die jedoch kulturell gar nicht nicht als >wahr< angesehen werden können. Krankheit in diesem Sinne ist die Domäne des biomedizinischen Denkstils und der ihn charakterisierenden Methoden. Davon grenzt Kleinman zweitens den Begriff der illness ab, womit er das Kranksein einer Person bezeichnet. Hierunter fasst er vor allem die je individuellen Wahrnehmungen von und Erfahrungen mit bestimmten, sozial oder kulturell als »nicht-gesund « markierten Zuständen, die eine Person macht. Diese Zustände müssen dabei nicht notwendigerweise in die biomedizinische Kategorie des Pathologischen fallen, und wenn sie es tun, können sie ggf. ganz anders interpretiert werden, als dies ein Mediziner vor dem Hintergrund seines »objektiven Wissens« täte. Disease und illness sind daher nicht miteinander identisch; beide zusammen ergeben aber einen Phänomenbereich, den Kleinman drittens als sickness bezeichnet. Für Kleinman sind Anthropologen vor allem für den Bereich der illness - also der kulturell geprägten Krankheitswahrnehmungen - zuständig.

Kranksein im Kleinman'schen Sinne ist hochgradig abhängig von sozial und kulturell differenten »Erklärungsmodellen« (explanatory models), die Konzepte über die Entstehung von Erkrankungen, Vorstellungen über den Krankheitsverlauf und über die angemessene Behandlung des Zustandes umfassen. Diese Modelle ordnen das Krankheitserleben in einer spezifischen, kulturell geprägten Weise und versehen es mit einer Bedeutung - sie bilden Realität ab. Aber darü-

15 Arthur Kleinman/Leon Eisenberg/Byron J. Good: »Culture, Illness, and Care Clinical Lessons from Anthropologic and Cross-Cultural Research«, in: Annals of Internal Medicine 88 (1978), S. 251-258; vgl. auch Arthur Kleinman: Writing at the Margin. Discourse between Anthropology and Medicine, Berkeley 1995. 
ber hinaus legen sie auch Handlungsweisen nahe, lassen andere als ungünstig erscheinen und - indem körperlicher Erfahrung ein spezifischer Sinn verliehen wird - erzeugen selbst subjektive wie intersubjektive Realität. »Explanatory models « sind Modelle des Krankheitsgeschehens, zugleich aber auch Modelle dessen, wie Kranke und ihr soziales Umfeld reagieren sollen, was angemessene Formen des Leidens an diesen körperlichen Zuständen sind und wie man in die Welt sowie den eigenen Körper mit welchen Mitteln eingreifen soll. Die Quelle dieser »explanatory models« wird dabei in den »Kosmologien« von Gesellschaften verortet, also in ihrer Kultur und im Raum der sozialen Interaktionen. ${ }^{16}$

In der Medizinanthropologie ist diese Unterscheidung zwischen disease und illness weitgehend unstrittig, nachteilig ist jedoch, dass dadurch die medizinische Definition von Krankheit als objektiv und das Erleben der Krankheit als subjektiv und kulturell geformt dargestellt wird. Damit wird verkannt, dass auch die Medizin als soziales (Sub-)System und als geprägt von kulturellen, historisch variablen Vorannahmen interpretiert werden muss, das »explanatory models « eigener Art hervorbringt. Die Anthropologen Allan Young ${ }^{17}$ und Ronald Frankenberg $^{18}$ haben deshalb bereits in den 1980er Jahren eine modifizierte Sicht vorgeschlagen. Die Unterscheidung von disease (Krankheit als Bezeichnung für wissenschaftlich-pathologische Modelle) und illness (Kranksein als Bezeichnung für die individuelle Wahrnehmung von Krankheit) wird beibehalten; aber sickness (Erkrankung) soll nun nicht mehr nur ein Sammelbegriff sein, unter den disease und illness fallen, sondern er wird spezifisch als eine dritte Dimension gefasst. Er bezeichnet jene sozialen Prozesse, durch die illness und disease sozialisiert werden. Etwa dadurch, dass beunruhigenden Verhaltensweisen oder physischen Phänomenen eine Bedeutung zugewiesen wird, sie also in Symptome übersetzt werden, aus denen wiederum spezifische Handlungen (therapeutische Interventionen, angemessene Formen des Leidens oder die Äußerung von Mitgefühl) fol-

16 Dies unterscheidet Kleinman deutlich von allen Ansätzen, die sich dem Problem des Krankheitserlebens von einer individualistischen Perspektive her nähern, vor allem natürlich von psychologisch informierten Ansätzen. Im Gegensatz dazu ist für die Medizinethnologie der Aufmerksamkeitsfokus stets auf die kulturellen Muster, den Lebensstil einer Gruppe, das alltägliche Handeln sowie die leitenden Vorstellungen und Werte von Menschen - ihre Kosmologien und Handlungsmuster in Aktion, im täglichen Vollzug - gerichtet.

17 Allan Young: »The Anthropology of Illness and Sickness«, in: Annual Review of Anthropology 11 (1982), S. 257-285.

18 Ronald Frankenberg: »Medical anthropology and development: a theoretical perspective«, in: Social Science \& Medicine 14 (1980), S. 197-207. 
gen. Der Begriff »sickness « historisiert und kontextualisiert sowohl wissenschaftlich-pathologische Modellvorstellungen wie auch die Muster der Krankheitserfahrungen; und die Analyse derjenigen Prozesse, die zu sickness führen, klärt dann vor allem die Frage, wer aus welchen Gründen wann an welcher Krankheit wie leidet. ${ }^{19}$

\section{Wie Soziales UND KULtUR >UNTER DiE HAUT GEHT،}

Im Folgenden werden einige Beispiele dafür vorgestellt, wie in der Geschichte der Medizin sickness produziert wurde; damit soll verdeutlicht werden, wie die von der Medizin seit langer Zeit gehegte Intuition, dass Soziales und Kulturelles >unter die Haut geht<, in konkrete ätiologische Modelle umgesetzt wurde. Deutlich wird dabei auch, wie hierbei dominante Vorstellungen sozialer Ordnung (an der man leiden kann) und individuelle Handlungsanweisungen zur Krankheitsprävention ko-produziert wurden. Hiermit ist zugleich die stärkere These einer »downward causation « angelegt, bei der physikalistische Erklärungen von Krankheitsentstehung irritiert werden. ${ }^{20}$

Die Idee, dass Soziales und Kulturelles >unter die Haut geht< und krankmachen kann, wurde bereits im 17. Jahrhundert recht präzise anhand von einigen Fallgeschichten durch den Schweizer Arzt Johannes Hofer herausgearbeitet, eine Studie, die allerdings durch die im 18. Jahrhundert einsetzende Verwissenschaftlichung der Medizin dem institutionellen Vergessen anheimgegeben wurde. Hofer legte seine Studie als Dissertation 1688 in Mühlhausen vor; ${ }^{21}$ zentrales Thema der darin versammelten Fallgeschichten sind medizinisch relevante Effekte von sozialen und kulturellen Fremdheitserfahrungen. Hofer schildert den Fall eines gesunden jungen Mannes, der für sein Studium aus seinem Heimatkanton Bern nach Basel gegangen war. Dort habe er seit seiner Ankunft an Traurigkeit

19 Vgl. etwa die grundlegende Studie von Irving K. Zola zu unterschiedlichen Krankheits- und Schmerzwahrnehmungen bei Angehörigen der amerikanischen Unter- und Mittelschicht bzw. bei unterschiedlichen Einwanderergruppen: Irving K. Zola: »Culture and Symptoms - An Analysis of Patients' Presenting Complaints«, in: American Sociological Review 31 (1966), S. 615-630.

20 Vgl. hierzu etwa Jaegwon Kim: »Emergence: Core ideas and issues«, in: Synthese 151 (2006), S. 547-559; Ana M. Soto/Carlos Sonnenschein/Paul-Antoine Miquel: »On physicalism and Downward Causation in Developmental and Cancer Biology«, in: Acta Biotheoretica 56 (2008), S. 257-274.

21 Johannes Hofer: Dissertatio Medica de Nostalgia oder Heimwehe, Basel 1688. 
gelitten und erkrankte schließlich an einem - wie er selbst sagte - kontinuierlichen, aber nicht brennenden Fieber. Er litt an Herzschmerzen und weiteren, immer gavierenderen Beschwerden, sodass seine Bekannten schließlich mit seinem baldigen Tod rechneten und beschlossen, ihn nach Hause zu bringen, damit er dort bei seinen Verwandten sterben könne. Aber sobald er die Reise angetreten hatte - so Hofer -, besserte sich sein Zustand von Kilometer zu Kilometer. Als er Bern erreichte, sei er fast völlig wieder hergestellt gewesen. Hofer schildert noch weitere Fälle, in denen todkranke Patienten, die etwa als Hausmädchen fern der Heimat Dienst tun mussten, spontan von ihren Beschwerden geheilt wurden, sobald sie wieder zu Hause waren.

Hofer findet für diese Beschwerden eine für ihn und offenbar auch seine Zeitgenossen plausible Erklärung, das heißt, er transformiert sie in Symptome: Wenn gesunde Menschen durch ihr Schicksal aus ihrer vertrauten sozialen Umgebung gerissen würden, ja selbst wenn sie freiwillig ihre Heimatgemeinde verließen, seien sie oft nicht in der Lage, sich an die fremden Sitten und Gebräuche anzupassen. In Johannes Hofers Zeit konnten solche Anpassungsprobleme offenbar schon durch die räumliche, soziale und kulturelle Distanz zwischen den Städten Bern und Basel ausgelöst werden. Als Folge dieser Fremdheitserfahrung - so Hofer - träumten die Betroffenen immer öfter von ihrer alten Heimat, wurden, was wir heute als melancholisch bezeichnen würden, und verfielen dann in diese merkwürdige Krankheit, für die er den wissenschaftlichen Begriff der Nostalgia vorschlug - eine griechische Anleihe, mit der er das im volkstümlichen Wissen längst etablierte Syndrom des Heimwehs belegte.

Was lässt sich aus einer 1688 auf Latein veröffentlichten Dissertation lernen, die inzwischen ausschließlich Medizinhistoriker interessiert? Das von Hofer bearbeitete Phänomen - nämlich die Frage, welche körperlichen Effekte eine grundlegende Fremdheitserfahrung haben kann, und sein ätiologisches Modell der Nostalgia - impliziert eine Konzeption sozialer und kultureller Ordnung, deren Nachhall in populären Vorstellungen noch immer präsent ist: Es ist ein statisches Konzept ständisch organisierter sozialer Ordnung, in der jeder einen festen Platz in einem weitgehend unbeweglichen Netz sozialer Beziehungen einnimmt. Und es ist ein territorialisiertes Konzept kultureller Ordnung, bei dem Heimat Eingebundenheit in eine spezifische Landschaft und die Bindung an lokal tradierte Sitten und Gebräuche meint. Pointiert gesagt, sind Hofers Patienten sozial und territorial gleichsam unverrückbar - werden sie in einen neuen sozialen, kulturellen oder landschaftlichen Zusammenhang verpflanzt, folgt unausweichlich Krise und Krankheit. Heilung ist erst möglich, wenn ihre soziale, kulturelle und territoriale Ökologie wieder restauriert wird, auf die jeder Einzelne schicksalhaft verwiesen ist. Hier wird soziale und räumliche Mobilität zum Risiko, weil 
die natürliche Ordnung des Sozialen - und der natürliche Platz des Einzelnen darin - verletzt wird.

Es ist durchaus bemerkenswert, dass eine solche Vorstellung sozialer und territorialer Verortung, wie sie etwa immer noch in der rhetorischen Figur der »entwurzelten Migranten « präsent ist, auch in der Gegenwart nicht nur populäre Diskurse prägt, sondern auch implizit viele Hintergrundannahmen gegenwärtiger klinischer Diskussionen um den Zusammenhang von Migration und Gesundheit: »Entwurzelung « wird immer noch für eine Vielzahl gesundheitlicher und sozialer Pathologien verantwortlich gemacht. ${ }^{22}$ Doch so verbreitet diese Intuition ist, so wenig wird die Frage des Zusammenhanges von sozialer Ordnung, der Rolle des Individuums in ihr und Gesundheit explizit problematisiert. Dabei sind solche »Fremdheitserfahrungen« durchaus nicht nur im Migrationskontext bedeutsam, wie sie von Hofer erstmals beschrieben wurden. »Fremdheit« und dadurch ausgelöste gesundheitliche Belastungen können - folgt man etwa einer neueren britischen epidemiologischen Studie - auch durch nicht überbrückbare soziale Distanz erzeugt werden. Interessant ist diese Studie aber vor allem unter dem Aspekt der Ko-Konstruktion des Sozialen und des Individuums: Anders als bei Hofer wird hier keine vormoderne, ständische Ordnung naturalisiert, sondern das Soziale - im Einklang mit der Kosmologie der Moderne - als Raum der Entfaltung der natürlichen Individualität des Einzelnen verstanden - ein Raum allerdings, der durch zahlreiche Schranken und Exklusionsmechanismen charakterisiert ist.

Ausgangspunkt der Studie von Simon Charlesworth und seinen Mitarbeitern ist der Befund, dass seit den 1980er Jahren die Unterschiede in der Lebenserwartung zwischen der Mittelschicht und der sozialen Unterschicht in Großbritannien dramatisch zunehmen. ${ }^{23}$ Lebten Angehörige der britischen Mittelschicht in den 1970er Jahren noch ca. fünf Jahre länger als Unterschichtangehörige, betrug die Differenz Mitte der 1990er Jahre bereits neun Jahre für Männer und sechseinhalb Jahre für Frauen. Hierfür machen die Autoren vor allem psychosoziale Mechanismen verantwortlich - etwa den dramatischen Verfall der Lebensbedingungen vor allem der schlecht ausgebildeten Arbeiter, die Auflösung des sozialen $\mathrm{Zu}$ sammenhalts, den Zusammenbruch der familiären Unterstützungsmechanismen, ein schwindendes Gefühl, über das eigene Leben Kontrolle zu haben, soziale Ausgrenzungsprozesse etc.

22 Vgl. etwa Eckhardt Koch/Matthias J. Müller: »Migration associated stress factors for depression«, in: Nervenarzt 78 (2007), S. 485-485.

23 Simon J. Charlesworth/Paul Gilfillan/Richard Wilkonson: »Living inferiority«, in: British Medical Bullettin 69 (2004), S. 49-60. 
Am Beispiel eines jungen Mannes aus der Unterschicht, der über den zweiten Bildungsweg kommend an seiner lokalen Universität studierte, um schließlich in den Polizeidienst einzutreten, erläutern sie, dass schon der Weg an die Universität gleichsam einen Passionsweg für ihn darstellte - in seinen Worten:

»Es zermürbt dich langsam, wenn du da jeden Tag hinmusst. Wenn du hinfährst, gibt es einen Punkt, an dem du merkst >hier fängt die Uni an<. Und ich fühle, wie sich mein Magen zusammenzieht, es geht ab diesem Punkt bergab. Und auf dem Rückweg ist es an diesem Punkt, wo du wieder nach Hause kommst, hier fängt die Zone an, in der du dich wieder wohlfühlen kannst. Ich merke das ganz deutlich: >Ich bin raus aus der Uni, ich fühle mich wieder wohl, ich komme mit der Situation wieder zurecht.< Und deshalb fahre ich auch zwischen den Seminaren immer wieder nach Hause, nur um dieses Gefühl zu erleben, dass es mir wieder gut geht. ${ }^{24}$

Es sind nicht die intellektuellen Anforderungen der Universität, die bei ihm dieses Unwohlsein auslösen, sondern die Konfrontation mit den Kommilitonen, die überwiegend aus der Mittelschicht entstammen. Es sind ihre - wie er es wahrnimmt - abschätzigen Blicke, die Erfahrung, dass er als Angehöriger der Unterschicht nicht ernst genommen wird, dass sich seine Studienkollegen über seinen Dialekt lustig machen, seinen Kleidungsstil belächeln, sich über sein Wohnviertel abschätzig äußern. Aber auch umgekehrt gilt, dass er den Lebensstil seiner Mitstudenten als völlig fremd wahrnimmt, ihre Haltung zur Welt und ihre Werteinstellungen nur schlecht nachvollziehen kann. Vor allem aber ist es die Erfahrung, dass er nicht über die notwendigen sozialen und kulturellen Kompetenzen verfügt, die man braucht, um sich sicher und selbstbewusst im universitären Milieu zu bewegen.

Quasi spiegelbildlich zu Hofers Vorstellung einer natürlichen sozialen Ordnung wird bei Charlesworth et al. die Ordnung der Klassengesellschaft als unnatürliche, vor allem aber ungerechte Beschränkung des natürlichen sozialen Mobilitätsbestrebens gedeutet: Das Spiel der Distinktion und der »feinen Unterschiede « - um einen Begriff Pierre Bourdieus aufzunehmen -, mit dem kulturelle und soziale Dominanz der oberen Schichten durchgesetzt wird, sorgt für eine chronische soziale, geistige und schließlich gesundheitliche Belastung der »have-nots«. Hier wird ein ungerechtes und auf Exklusion setzendes Gesellschaftssystem zum Risikofaktor. Epidemiologisch ist der Befund, dass es eine kausale Beziehung zwischen sozialer Isolation, Ausgrenzung, Depression und mangelnder Unterstützung durch ein soziales Umfeld und kardiovaskulären Er- 
krankungen gibt, schon seit vielen Jahren sehr gut belegt. So wird »Einsamkeit ${ }^{25}$ als mindestens ebenso großer Risikofaktor für Herzinfarkte wie Rauchen, hoher Blutdruck oder erhöhte Blutfettwerte angesehen. Ähnlich argumentieren der Medizinsoziologe Johannes Siegrist und Michael Marmot, einer der führenden Epidemiologen, auf Grundlage einer in mehreren europäischen Ländern durchgeführten Studie. ${ }^{26}$ Für sie sind es vor allem die Wirkungen - wie sie es nennen - »ungünstiger psychosozialer Umwelten« - etwa hohe Anforderungen bei geringer Selbstbestimmung am Arbeitsplatz oder ein dauerhaftes Missverhältnis zwischen hoher Leistung und geringer Belohnung, die langfristige, negative Gesundheitseffekte erzeugen. Zahlreiche, groß angelegte epidemiologische Studien - etwa die Framingham $-{ }^{27}$ oder die Whitehall-Studie ${ }^{28}$ - produzieren eine so schlagende Evidenz dieser sozialen Effekte auf die Gesundheit, dass Richard Eckersley, einer der weltweit führenden Epidemiologen in einem Überblicksartikel nur noch rhetorisch fragen konnte: »Is modern Western culture a health hazard? ${ }^{29}$

Vor dem Hintergrund dieser sich immer mehr verdichtenden Befunde der Epidemiologie und der neueren public-health-Forschung ist es bemerkenswert, dass sich die Sozialwissenschaften doch allmählich und sehr vorsichtig auf das von ihnen als durch biologistische Fallstricke überzogene Parkett zu wagen beginnen. Eine wichtige Motivation ist dabei nicht zuletzt die Wahrnehmung, dass in den Naturwissenschaften nur eingeschränkte methodische wie theoretische Kompetenz vorliegt, mit Ungleichheitsphänomenen jenseits einfacher sozialökonomischer Indikatorenforschungen umgehen zu können. So plädiert etwa Martin Diewald in einem kürzlich in der Zeitschrift für Soziologie veröffentlichten Artikel für einen intensiveren Austausch der Soziologie mit der Epidemiologie oder der Verhaltensgenetik, um das »Zusammenspiel genetischer Prädisposi-

25 Anselm Eder: »Risk factor loneliness. On the interrelations between social integration, happiness and health in 11-, 13- and 15-year old schoolchildren in 9 European countries«, in: Health Promotion International 5 (1990), S. 19-33.

26 Johannes Siegrist/Michael Marmot: »Health inequalities and the psychosocial environment— two scientific challenges«, in: Social Science \& Medicine 58 (2004), S. $1463-1473$.

27 William B. Kannel/Daniel L. McGee: »Diabetes and cardiovascular disease. The Framingham study«, in: JAMA, 241, 19 (1979), S. 2035-2038.

28 Hans Bosma et al.: »Low job control and risk of coronary heart disease in Whitehall II (prospective cohort) study«, in: British Medical Journal 314 (1997), S. 558-565.

29 Richard Eckersley: »Is modern Western culture a health hazard?«, in: International Journal of Epidemiology 35 (2006), S. 252-258. 
tionen mit sozialen Einflüssen über die Lebenszeit und die historische Entwicklung von Populationen hinweg « unter Rückgriff auf soziologischen Sachverstand aufzuklären. ${ }^{30}$ Es bleibt abzuwarten, welches Engagement die Soziologie hier künftig entwickeln wird.

\section{PSYCHOSOMATIK, GESPROCHEN MIT AMERIKANISCHEM AKZENT: STRESS}

Natürlich hat die oben zitierte Intuition Eckersleys, dass die moderne Kultur den Menschen krankmache, eine lange zivilisationskritische Tradition. Ein prominenter und im weiteren medizinischen Feld immer noch einflussreicher Zweig ist sicherlich die seit den 1920er Jahren in Deutschland eng mit den Arbeiten Viktor von Weizsäckers, Georg Groddecks und Felix Deutschs verbundene »Psychosomatische Medizin«. Für Anne Harrington, die 2008 ihre beeindruckende Geschichte der »Geist-Körper Medizin« vorlegte, grenzt sich die Psychosomatische Medizin deutscher Prägung unter Rückgriff auf Impulse des holistischen Denkens des späten 19. Jahrhunderts klar gegen eine als reduktionistisch erfahrene, von naturwissenschaftlichem, insbesondere biologischem Denken geprägte Medizin $a b .{ }^{31}$ Die tendenziell »anti-szientistische«, unter anderem von der Romantik beeinflusste Haltung dieser Tradition der Psychosomatik macht dieses Erklärungsangebot jedoch kaum anschlussfähig an biomedizinische Erklärungsmodelle von Krankheit.

Anders ist dies in der Spielart der Psychosomatik, die seit den 1920er Jahren mit >amerikanischem Akzent< zu sprechen beginnt. Es sind insbesondere die grundlegenden Arbeiten des US-amerikanischen Physiologen Walter B. Cannon, der einen biochemisch-neurologischen Mechanismus postulierte, über den Soziales physiologische Wirkungen entfalten konnte. Für seine Erklärung griff Cannon auf seine vor allem in Tierexperimenten in den Laboratorien der Harvard Medical School gewonnenen empirischen Daten zur Physiologie der Schockreaktion und zu körperlichen Reaktionen bei starken Emotionen wie Schmerz, Angst, Zorn und Hunger zurück. Die von ihm vertretene These lautete: Starke

30 Martin Diewald: »Zur Bedeutung genetischer Variation für die soziologische Ungleichheitsforschung «, in: Zeitschrift für Soziologie 39 (2010), S. 4-21, hier S. 16.

31 Anne Harrington: The cure within: a history of mind-body medicine, New York 2008, S. $88 f$. 
emotionale Erregungen können die Gleichgewichts-Mechanismen des Körpers so nachhaltig stören, dass die Betroffenen sogar daran sterben können. ${ }^{32}$

Die von Cannon vorgeschlagene Perspektive auf somatische Regelungssysteme hat sich als außerordentlich fruchtbar erwiesen; in seiner Tradition wird in den letzten Jahren verstärkt das komplexe Zusammenspiel des neuroendokrinen, des kardiovaskulären und des Immunsystems untersucht. Unter dem Begriff der allostatischen Anpassung ${ }^{33}$ wird der Frage nachgegangen, wie der Körper auf externe Stimuli oder die Veränderung von Umweltbedingungen reagiert. Die zugrunde liegende Modellvorstellung ist dabei, dass auf länger wirkende Belastungen - sozialer Stress, Infektionen oder Nahrungsmittelknappheit - verschiedene körpereigene Systeme so ineinandergreifen, dass eine langfristige Anpassungsreaktion an die negativen Umweltreize ausgelöst wird. ${ }^{34}$ Im Gegensatz zu den von Cannon beschriebenen, unmittelbar der Lebenserhaltung dienenden Regelkreisen, die hoch dynamisch um einen »Normalwert « schwanken - wie etwa beim Blutdruck oder der Körpertemperatur -, werden bei allostatischen Reaktionen auf chronischen Stress diese Normwerte im belasteten Körper dauerhaft verschoben: Der Körper muss gleichsam einen Preis zahlen, wenn er sich langfristig an ungünstige psychosoziale oder physische Situationen anpassen muss. ${ }^{35}$

32 Vgl. hierzu ausführlich Walter B. Cannon: Der Weg eines Forschers. Erlebnisse und Erfahrungen eines Mediziners, München 1945. Vgl. als wissenschaftshistorische Einordnung der Bedeutung Cannons Jakob Tanner: »)Weisheit des Körpers $<$ und soziale Homöostase. Physiologie und das Konzept der Selbstregulation«, in: Philipp Sarasin/Jakob Tanner (Hg.), Physiologie und industrielle Gesellschaft. Studien zur Verwissenschaftlichung des Körpers im 19. und 20. Jahrhundert, Frankfurt/M. 1998, S. 129-169.

33 Peter Sterling/Joseph Eyer,: »Allostasis: A new paradigm to explain arousal pathology«, in: James Reason/Shirley Fischer, Handbook of Life Stress, Cognition and Health, New York 1988, S. 629-649.

34 Dieser Zusammenhang wurde erstmals beschrieben von Hans Selye: »Syndrome produced by diverse nocuous agents«, in: Nature 138 (1936), S. 32. Selyes Studien machten den Begriff »Stress« populär und begründeten eine Forschungsrichtung, die biografische Akkumulationen belastender Einflüsse auf körperliche Zustände beschrieb; vgl. Hans Selye: The Stress of Life, New York 1956.

35 Vgl. Bruce S. McEwen: »Protective and damaging effects of stress mediators: central role of the brain«, in: Biological Basis for Mind Body Interactions 122 (2000), S. 2534; Bruce S. McEwen/John C. Wingfield: »The concept of allostasis in biology and biomedicine«, in: Hormones and Behavior 43 (2003), S. 2-15, hier S. 3. 
Aus einer sozialwissenschaftlichen Perspektive ist interessant, dass mit dieser Theorie der akkumulierten Belastungen eine körperbiografische Perspektive eröffnet wird: Wie alle kybernetischen Systeme weise auch der Körper einen Memo-Effekt auf, indem etwa sozialer Stress - etwa ein geringer sozialer Status am Arbeitsplatz, Existenzängste durch drohenden Arbeitsplatzverlust oder auch ein ungünstiges Wohnumfeld - das individuelle Risiko für die Entwicklung chronischer Krankheitszustände bedeutend erhöhen können. ${ }^{36}$ Ging es bei Cannon noch um das Problem der Erhaltung körperlicher Stabilität angesichts kurzfristig wirkender Umwelt-Turbulenzen, geht es in neueren Studien zu chronischen Belastungen zugespitzt um die Frage, ob man etwa an geringem sozioökonomischen Status oder an durch soziale Exklusion bedingtem, biografisch akkumuliertem Stress erkranken oder - früher - sterben kann.

Stress erscheint in neueren Studien als Auslöser sehr unterschiedlicher pathologischer Effekte: als Grund für Fehlgeburten oder Angststörungen, als Faktor, der Dickleibigkeit fördert oder cardiovaskuläre Erkrankungen. Diese Aufzählung soll nicht fortgesetzt werden, wichtig ist nur, dass in allen diesen Fällen ein enger, systemischer Zusammenhang von neurologischen, immunologischen und physiologischen Effekten in Reaktion auf soziale Umwelteinflüsse postuliert wird. Folgt man der Argumentation von McEwen und anderen Stressforschern, dann werden durch das Stress-Modell belastende Vergangenheiten in der Gegenwart aktualisiert: Sie prägen das Spektrum, in dem der Körper reagieren kann. Der Entwicklungsbiologe René Dubos schlug bereits Mitte der 1960er Jahre für solche langfristigen physiologischen Prägungen den Begriff »biological Freudianism « vor. $^{37}$ Allerdings war Mitte der 1960er Jahre noch kein biologischer Mechanismus erkennbar, über den Umwelteffekte langfristige pathologische Verschiebungen in den körperlichen Reaktionsmustern auslösen konnten; in den letzten Jahren scheinen nun epigenetische Modelle eine Lösung dieses (alten) Rätsels anzubieten. Wichtig im hier diskutierten Zusammenhang sind diese Modelle auch deshalb, weil sie dazu führen können, die Bedeutung des »risk thinking « (N. Rose) im Alltag zu erhöhen: Der nahegelegte »präventive Lebensstil« fordert zur generalisierten Vorsicht auf, er aktualisiert potenzielle gesund-

36 Vgl. Bruce S. McEwen: »Protective and damaging effects of stress mediators«, in: New England Journal of Medicine 338 (1998), S. 171-179; Bruce S. McEwen: »Allostasis and allostatic load: Implications for neuropsychopharmacology«, in: Neuropsychopharmacology 22 (2000), S. 108-124.

37 René Dubos/Dwayne Savage/Russell Schaedler: »Biological Freudianism - Lasting Effects of Early Environmental Influences«, in: Pediatrics 38 (1966), S. 789-800. 
heitliche Zukünfte in der Gegenwart, wenn das Alltagshandeln zunehmend durch die Vermeidung schädlich erachteter Wirkungen bestimmt wird.

\section{Epigenetische Revisionen}

Ende der 1990er Jahre nahm eine schwedische Forschungsgruppe die Fragerichtung des »biologischen Freudianismus« auf. Die Gruppe untersuchte, ob Schwankungen in der Nahrungsmittelversorgung bei Heranwachsenden Auswirkungen auf die Entwicklung der Keimzellen haben könnten und ob damit ein Mechanismus für die nicht-genetische - also epi-genetische - Weitergabe dieser Effekte wahrscheinlich sei. ${ }^{38}$ In einer Studie erfassten die Forscher die Gesundheitsbiografien von 300 Personen, die in den Jahren 1890, 1905 und 1920 in einem nordschwedischen Bezirk geboren worden und aufgewachsen waren, und verknüpften diese Daten mit sozialhistorischen Befunden, aus denen auf den Ernährungszustand der jeweiligen vier Großeltern und der beiden Eltern jedes Probanden rückgeschlossen werden konnte. Für die fraglichen Zeiträume im 19. Jahrhundert waren dabei mehrere gravierende Hungersnöte in der Region zu verzeichnen, denen eine Reihe von $>$ fetten $<$ Jahren gegenüberstanden.

Im Ergebnis konnte gezeigt werden, dass Knappheits- oder ÜberflussPerioden in der Nahrungsversorgung der Großeltern einen deutlich feststellbaren Effekt auf das Risiko der Enkelgeneration hatten, an Diabetes II oder kardiovaskulären Erkrankungen zu leiden. War etwa der Großvater in seiner Jugendzeit von einer Hungersnot betroffen, wies der Enkel ein deutlich reduziertes Diabetes-Risiko auf; war der Großvater hingegen in Zeiten eines Nahrungsüberflusses aufgewachsen, dann wiesen die Enkel ein bis zu vierfach erhöhtes Risiko auf, an Diabetes zu erkranken. Die genetische Ausstattung der untersuchten Personen erscheint in dieser Studie nicht mehr als inerte Substanz, die unverändert von Generation zu Generation weitergegeben wird, sondern sie ist durch epigenetische Effekte in ihrer Funktionsweise zeitlich und kontextuell geprägt. Das Genom - also die gesamte genetische Information eines Organismus - erweist sich als gegenüber Umwelteinflüssen sensibles >Organ $<$ mit intergenerationeller Erinnerung und es ist durch Umwelteinflüsse langfristig prägbar, indem bestimmte Sequenzen ein- oder ausgeschaltet werden. Dies ist der Fall in unterschiedlichen Zelltypen, die jeweils andere Funktionen im Organismus ausführen, aber auch

38 Gunnar Kaati/Lars O. Bygren/Sören Edvinsson: »Cardiovascular and diabetes mortality determined by nutrition during parents' and grandparents' slow growth period «, in: European Journal of Human Genetics 10 (2002), S. 682-688. 
dann, wenn Umwelteinflüsse die Funktionsweise genetischer Informationen beeinflussen, ein Effekt, der teilweise von Generation zu Generation weitergegeben, also epigenetisch vererbt werden kann. ${ }^{39}$ Die Summe dieser Gen-Zustände, die durch Ein- oder Abschalten erzeugt wird, wird als »Epigenom« bezeichnet.

Mosche Szyf, Epigenetiker an der McGill University in Montreal, formuliert den aktuellen Forschungsstand wie folgt:

»Im Gegensatz zum Genom ist das Epigenom [...] relativ dynamisch und wird durch verschiedene Umweltfaktoren [vor allem durch Toxine und Ernährungsmuster, SB] während der fetalen und frühen postpartalen Entwicklung beeinflusst. Zwar ist die Mehrzahl der epigenetischen Markierungen während des Lebens wahrscheinlich stabil, ein Teil bleibt aber dynamisch. $\ll^{40}$

Diese epigenetische Plastizität betrifft auch die Regulation von komplexen Verhaltensmustern, wie an bahnbrechenden Experimenten mit Ratten gezeigt werden konnte, bei denen ein gestörtes Brutpflegeverhalten der Elterntiere die Genexpression und Stressresistenz bei ihren Jungtieren nachhaltig veränderte. ${ }^{41}$ In allen Fällen sind epigenetische Mechanismen für diese auch intergenerationell vererbbaren Verhaltensunterschiede verantwortlich. ${ }^{42}$

Diese epigenetischen Mechanismen sorgen dafür, dass Umwelten tatsächlich > unter die Haut gehen<; hiermit wird ein sehr mächtiges Modell etabliert, das es zu erlauben scheint, eine ganze Reihe von bislang biochemisch nicht erklärbaren Phänomenen zu deuten. ${ }^{43}$ Es werden Zusammenhänge sichtbar gemacht - also: in einer spezifischen Form repräsentiert -, aus denen zugleich veränderte Eingriffs- und Interventionsstrategien in mögliche pathogene Zustände folgen. Dies

39 Barbara McClintock: »The Significance of Responses of the Genome to Challenge«, in: Science 226 (1984), S. 792-801; vgl. auch Rudolf Jaenisch/Adrian Bird: »Epigenetic regulation of gene expression: how the genome integrates intrinsic and environmental signals«, in: Nature Genetics Supplement 33 (2003), S. 245-254.

40 Moshe Szyf: » Dynamisches Epigenom als Vermittler zwischen Umwelt und Genom«, in: Medizinische Genetik 21 (2009), S. 7-13, hier S. 7.

41 Vgl. Moshe Szyf/Ian Weaver/Michael Meaney: »Maternal care, the epigenome and phenotypic differences in behavior «, in: Reproductive Toxicology 24 (2007), S. 9-19.

42 Vgl. etwa Anne C. Ferguson-Smith/Mary-Elizebeth Patti: »You Are What Your Dad Ate«, in: Cell Metabolism 13 (2011), S. 115-117; Michael K. Skinner: »Metabolic Disorders Fathers' Nutritional Legacy«, in: Nature 467 (2010), S. 922-923.

43 Vgl. hierzu Jörg Niewöhner: »Epigenetics: Embedded bodies and the molecularisation of biography and milieu«, in: BioSocieties, advance online publication 13.6.2011. 
lässt sich gut an gegenwärtigen Debatten der Gesundheitspolitik und der publichealth-Experten um die »Epidemie der Adipositas« erläutern. Hier ist es der Begriff des »obesogenic evironments $«{ }^{44}$ der momentan Karriere zu machen beginnt. Leitthese ist hier: Bisherige primär auf »Aufklärung « setzende, erzieherische oder pharmazeutische Maßnahmen, mit denen gegen die zunehmende Dickleibigkeit vor allem in den unteren Sozialschichten vorgegangen werden sollte, sind durchweg gescheitert, weil die Menschen vor allem in sozial benachteiligten Stadtteilen in Umwelten leben, in denen zu viel kalorienreiche Nahrung (sprich: Fast Food) und zu wenig Bewegungsmöglichkeiten verfügbar seien. Eine positive Verhaltensänderung sei nicht durch kognitive Interventionen, sondern nur durch eine radikale Veränderung des »food and nutrition environments $«{ }^{45}$ möglich.

Dies kann einerseits als Wiederauferstehung alter Setting-Ansätze gedeutet werden, in denen vorausgesetzt wird, dass vor allem sozial benachteiligte Menschen nicht über die notwendige Selbstkontrolle verfügen, einen gesunden Lebensstil zu realisieren. Fehlt der Wille oder die notwendige Möglichkeit zur erfolgreichen, effektiven »Selbsteinwirkung «, dann helfe nur fürsorgliche »Fremdeinwirkung«, externalisierte Kontrolle und eine Manipulation der Handlungsbedingungen. Vor dem Hintergrund epigenetischer Modelle werden diese Annahmen des Setting-Ansatzes nochmals zugespitzt. Denn nun lässt sich auch spekulieren, dass es möglicherweise epigenetische Mechanismen seien, die Menschen veränderungsresistent machten: Lebensstil-Veränderungen scheitern hier nicht nur am >schwachen Willen $<$, sondern an durch Umwelteinflüsse gebahnten körperlichen Reaktionsmustern. Gesundheitspolitische Brisanz erhält diese Feststellung dann noch zusätzlich durch den epigenetischen Befund, dass etwa der Lebensstil der Eltern den weiteren Lebensweg und die Gesundheit ihrer Kinder noch im Mutterleib zu prägen beginnt. Gesundheitspolitische Interventionen können hieraus neues legitimatorisches Kapital schlagen - neben den neoliberalen Anspruch, dass Bürger zu einer Sorge um sich verpflichtet seien, tritt hier die neo-paternalistische Sorge um die nächsten Generationen.

44 Garry Egger/Boyd Swinburn: »An >ecological< approach to the obesity pandemic «, in: British Medical Journal 315 (1997), S. 477-480; Boyd Swinburn/Garry Egger/Fezeela Raza: »Dissecting Obesogenic Environments: The Development and Application of a Framework for Identifying and Prioritizing Environmental Interventions for Obesity«, in: Preventive Medicine 29 (1999), S. 563-570.

45 Vgl. u. a. Amelia Lake/Tim Townshend: »Obesogenic environments: exploring the built and food environments «, in: The Journal of the Royal Society for the Promotion of Health 126 (2006), S. 262-267. 


\section{EPISTEMISCHE DialeKTIK UND DIE KO-KONSTRUKTION VON PATHOLOGIE UND SOZIALITÄT}

Mit etwas Distanz offenbart sich in den neueren Entwicklungen der Biomedizin ein dialektischer Prozess. So argumentieren etwa die beiden Wissenschaftshistoriker Staffan Müller-Wille und Hans-Jörg Rheinberger in ihrer jüngsten Publikation, dass es ausgerechnet die reduktionistischen Forschungsstrategien der Molekularbiologie und Gentechnologie waren, die zunehmend eine »systemischere Sicht auf das Funktionieren, die Entwicklung und die Evolution der Organismen« erforderlich gemacht habe. Diese Diagnose erinnert an Canguilhems oben zitierte, hellsichtige Analyse der in den physikalistischen Reduktionismus der Lebenswissenschaften eingebauten Dialektik: Die immer weitere Molekularisierung der Biologie erzwinge als Gegenbewegung eine systemischere Sicht, die es erlaube, die Einzelbefunde wieder theoretisch zu integrieren. Doch MüllerWille und Rheinberger sehen die gegenwärtige Tendenz zur systemischen Perspektive nicht als Gegentendenz, sondern sie ergebe sich nun quasi naturwüchsig aus den reduktiven Strategien der Molekularbiologie. Momentan werde diese Tendenz noch weiter beschleunigt, weil die in den letzten Jahren entwickelte Technologie der DNA-Chips es ermögliche, Aktivitätsmuster von Zellen in Realzeit zu erheben und gleichsam Schnappschüsse der Dynamik des zellulären Stoffwechsels bei unterschiedlichen Umweltbedingungen der Zellen zu erstellen. ${ }^{46}$ Im Gegensatz zum Holismus oder der Psychosomatik des frühen 20. Jahrhunderts, die eine ganzheitlichere Sicht auf den Organismus forderten, ohne jedoch entsprechende physiologische Mechanismen aufzeigen zu können, wie die Umwelt auf körperliche Prozesse einwirkte, wird nun eine neue Phase eingeleitet, die eine nicht-spekulative, biochemisch fundiertere Sicht auf KörperUmwelt-Kopplungen ermöglicht.

Begriffe wie Postgenomik, Epigenetik, Proteomik oder Organomik sowie der Versuch, zunehmend netzwerktheoretische oder emergenztheoretische Ansätze für die Modellbildung der Biologie einzusetzen, deuten an, dass es nicht mehr die Identifikation einzelner Gene, sondern deren multiple Funktion in dynamischen Stoffwechsel-Netzwerken ist, deren Verständnis zum leitenden Forschungsziel wird. Der Wissenschaftsphilosoph Lenny Moss fasst diese Tendenz prägnant unter dem Stichwort einer »Re-Kontextualisierung « des Genoms, das nun als offen gegenüber den inneren wie äußeren Milieus des Organismus ver-

46 Hans-Jörg Rheinberger/Staffan Müller-Wille: Vererbung. Geschichte und Kultur eines biologischen Konzeptes, Frankfurt/M. 2009, S. $271 \mathrm{f}$. 
standen werde. ${ }^{47}$ Auch diese Erkenntnis ist so neu nicht; schon in den 1980er Jahren definierten Biologen wie François Jacob ${ }^{48}$ oder Hans Mayr ${ }^{49}$ das Problem der biologischen Regulation von Organismen in wechselnden Umwelten als die zentrale Fragestellung der Biologie. Dabei argumentierten beide, dass sich Lebensprozesse nur unvollständig unter Rückgriff auf naturwissenschaftlichreduktionistische Theorien erklären ließen, weil Leben durch historische - individuelle wie kollektive - Entwicklung und Kontingenz ausgezeichnet ist.

Biologen wie Jacob, Mayr oder Dubos verwiesen damit auf die Bedeutung des Faktors Zeit - im Sinne evolutionärer, genealogischer wie biografischer Entwicklung - für die Erklärung biologischer Phänomene, ein Zusammenhang, den sie in den dominanten reduktionistischen Experimentalpraktiken nur unzureichend reflektiert sahen. Rheinberger und Müller-Wille weisen jedoch zu Recht auf das Spannungsverhältnis zwischen reduktionistischen Laborpraktiken und denen mit ihnen produzierten wesentlich komplexeren epistemischen Objekten und Ontologien hin - eine unvermeidliche Dialektik epistemischer Praktiken. Vor allem bei Theorien zur Krankheitsentstehung - also in jenen materielldiskursiven Praktiken, in denen sickness erzeugt wird - wird dieser Zusammenhang offensichtlich. Martyn Pickersgill hat dies in einem sehr lesenswerten, kürzlich veröffentlichten Artikel am Beispiel von Forschungen zu Persönlichkeitsstörungen in der Verhaltensgenetik und den Neurowissenschaften herausgearbeitet. In beiden Feldern werde innerhalb eines reduktionistischen Programms gearbeitet, aber die ontologischen Annahmen der konkreten Studien seien notwendigerweise alles andere als einfach:

»Die Entwicklung des Verhaltens und von Persönlichkeit wird als komplexer, multimodaler Prozess verstanden, der reziproke Interaktionen zwischen ganz unterschiedlichen Dimensionen des Biologischen und der Umwelt beinhaltet. Konzepte der Umwelt, wie sie von vielen Forschern angewandt werden, sind dabei weit gefasst, sie reichen von der zellulären Ebene bis zum sozio-ökonomischen Status. $\ll^{50}$

47 Lenny Moss: »From Representational Preformationism to the Epigenesis of Openness to the World? «, in: Annals of the New York Academy of Sciences 981 (From Epigenesis to epigenetics: The genome in context) (2002), S. 219-229.

48 François Jacob: Das Spiel der Möglichkeiten. Von der offenen Geschichte des Lebens, München 1983.

49 Ernst Mayr: Das ist Biologie. Die Wissenschaft des Lebens, Heidelberg, Berlin 1998.

50 Martyn Pickersgill: »Between Soma and Society: Neuroscience and the Ontology of Psychopathy«, in: Biosocieties 4 (2009), S. 45-60, hier S. 46. 
Allerdings sei zu konstatieren, dass die Modelle des Sozialen, die dabei von Biowissenschaftlern angewandt würden, oft extrem simpel und vereinfachend angelegt seien. Im Vergleich mit den Verständnissen sozialer Phänomene, wie sie in den Sozialwissenschaften entwickelt worden seien, kämen vor allem simplifizierte »Alltagskonzepte« des Sozialen zur Anwendung. Das entscheidende Argument von Pickersgill ist jedoch, dass Psychopathologien in diesem Forschungsparadigma nicht nur »neurologisiert«, sondern »die Psychopathen« im gleichen Schritt auch »ökologisiert« würden: Die Klassifikation von Individuen als »persönlichkeitsgestört « impliziere nicht nur, dass ihnen eine spezifische genetische und neurologische Disposition zugeschrieben werde, sondern eben auch, dass sie in einer Ökologie aus sozialen Strukturen, biografischen Erfahrungen und kulturell informierten Handlungsmustern situiert würden, Umweltfaktoren, die diese Dispositionen schließlich ins Pathologische transformierten.

Pickersgill spricht hier den oben unter dem Begriff der sickness diskutierten Zusammenhang an, nämlich jene sozialen Prozesse, in denen Krankheit und Kranksein in je historischen Konstellationen und unter spezifischen kulturellen Umständen bestimmt werden. Dabei ist offensichtlich, dass diese »Ökologisierung der Pathologie« stets auch normative und normalisierte Vorannahmen über Gesellschaft und die Rolle von Individuen in ihr implizieren. Aus einer sozialwissenschaftlichen Perspektive sollte deshalb präziser von einer Sozialisierung oder Kulturalisierung der Pathologie beziehungsweise von einer KoKonstruktion des Pathologischen und des Sozialen gesprochen werden. Dabei ist der Begriff der Konstruktion durchaus wörtlich zu nehmen, denn spätestens dann, wenn aus den Befunden etwa der Neurowissenschaften Handlungsvorschläge für Interventionen in Therapie und Prävention abgeleitet werden, entfalteten die vorgeschlagenen Erklärungsmodelle reale Wirkungen im Alltag nicht nur direkt betroffener Menschen. Und spätestens dann erweisen sich die von Naturwissenschaftlern angewandten, wenig komplexen Verständnisse des Sozialen als fatal, da aus Deskription oft - wohlmeinende - Präskription abgeleitet wird: moralisch aufgeladene Handlungsanleitungen für die Kranken und ihr soziales Umfeld. Abschließend soll skizzenhaft gezeigt werden, welche Vorstellungen des Sozialen und welche Konzepte des Individuums in den verschiedenen, oben vorgestellten Ätiologien impliziert sind. 


\section{KRANKHEIT, GESELLSCHAFT UND VERANTWORTLICHE INDIVIDUEN ALS WISSENSOBJEKTE}

In Johannes Hofers Dissertation über das Heimwehe wird implizit eine ständische, frühmoderne Sozialität mit fester territorialer Bindung der Menschen entworfen, eine Gesellschaft, deren Individuen von den zunehmend durch Bildungsoder Arbeitsmobilität erzeugten Anforderungen und Zumutungen überfordert sind. Es sind »sensible« Individuen, die jedoch mit kulturellen Fremdheiten schlecht umgehen können und deshalb an ihren angestammten Orten - räumlich und sozial - bleiben sollen. Im Gegensatz dazu sehen medizinische Experten die krankmachende Realität in den groß angelegten epidemiologischen Studien der 1950er und 1960er Jahre bis hin zu den zitierten neueren epidemiologischen Untersuchungen zu Effekten sozialer Exklusion durch ein Klassen- oder Schichtsystem charakterisiert, in dem Aufwärts- und Abwärtsmobilitäten Überforderungsoder Zumutungssituationen erzeugen, die den Menschen >unter die Haut gehen und Krankheitsrisiken analog zur sozialen Schichtung verteilen. Chronischer Stress gilt dabei als fast zwangsläufiges Ergebnis zivilisatorischen Fortschritts und eines auf Leistungsdruck programmierten sozialen Systems: Es ist die Moderne, die krankmacht, weil die Gesellschaft den >natürlichen< Bestrebungen der Individuen, sich zu entfalten, harte soziale Grenzen setzt. Zugleich aber wird den Einzelnen zugetraut - und zugemutet -, auf erkannte Risiken durch kluge Wahl alternativer Verhaltensweisen und eine Änderung des Lebensstils zu reagieren.

Im Gegensatz hierzu ist die neuere Diskussion der public-health-Experten durch ein gespaltenes Gesellschaftsmodell charakterisiert: Im gesellschaftlichen Oben regiert Wahlfreiheit von lebensstilistischen Orientierungen, die Individuen treffen Entscheidungen, auf die durch (Gesundheits-)Information und Aufklärung eingewirkt werden kann. Im gesellschaftlichen Unten dagegen werden Dispositionen oder Lebensstile vermutet, die durch einen fatalen Fatalismus oder Unausweichlichkeiten geprägt sind. Aber anders als bei den durch das Risikomodell geprägten Diskussionen um soziale Erkrankungswahrscheinlichkeiten kommt in neueren epidemiologischen Studien hinzu - verschärft durch epigenetische Befunde wie den oben erwähnten -, dass nun Individuen nicht mehr nur für ihr eigenes gesundheitliches Schicksal verantwortlich gemacht werden, sondern auch für das ihrer Kinder und Kindeskinder. Prävention erhält durch die zunehmend belegten epigenetischen Effekte, durch die Krankheitsdispositionen intergenerational vererbt werden können, aus Sicht der Gesundheitspolitik und -vorsorge noch größere Dringlichkeit. Hieraus - so kann spekuliert werden werden sich neue Eintritts- und Interventionspunkte für einen neuen Paternalismus ergeben, der sich nicht mehr nur in einer >fürsorglichen Belagerung< der 
Unterschichten mit wohlmeinenden Empfehlungen für gesundes Essen oder mehr Bewegung erschöpft, sondern sich durchaus zutraut und legitimiert sieht, korrigierend und disziplinierend ein- und durchzugreifen unter Berufung auf die Sicherung der Gesundheit nicht nur der gegenwärtigen, sondern auch der zukünftigen Generation. Dies jedoch ist Zukunftsmusik - eine, die nicht durch groBe Harmonien charakterisiert sein wird.

Für die Anthropologie ergibt sich hier - neben der selbstverständlichen Aufgabe, diese Entwicklungen ethnografisch in ihren Auswirkungen gerade auf gesellschaftlich marginale Gruppen genau zu beobachten, wie es gerade die internationale medical anthropology tut - noch eine weitere, nicht unwichtigere Aufgabe. Denn wenn die oben erläuterte These, dass Soziales und Kulturelles >unter die Haut gehen< kann, berechtigt ist, dann ist sozialwissenschaftliche Expertise nicht nur bei der Frage gefordert, wie betroffene Risikogruppen definiert werden können - und zwar nicht entlang simpler, höchst problematischer >ethnischer Klassifikationen, sondern trennschärfer nach möglichen LebensstilMerkmalen oder tatsächlichen Risikolagen. Noch wichtiger erscheint zudem, dass sich die Frage, was mögliche Mechanismen eines >unter-die-Haut-Gehens sein könnten, nur in einer engen transdisziplinären Zusammenarbeit zwischen Sozial- und Naturwissenschaften wird beantworten lassen. Nur so lassen sich wie man es noch reichlich tentativ nennen könnte - sozial und kulturell situierte Biologien und Pathologien erkennen und gegebenenfalls vermeiden. 